Note: This paper was submitted to CAOSP journal. 


\title{
Line of sight errors in eclipsing binary stars
}

\author{
A.S. Jayaram ${ }^{1}$ \\ Innovation and project consultancy services,Bangalore- 560040.India. \\ (E-mail: surya.jayaram@gmail.com) \\ India
}

Received: , ; Accepted: ?

\begin{abstract}
This paper will illustrate about the line of sight errors in eclipsing binary stars. Due to this error, the eclipsing binary stars will not be actually along be along the line of sight when the observer see them as eclipsing. This is due to longer time taken by the light to travel from the star at the far end to that of the star at the near end, with respect to the observer. That means, light from the star at the near end will reach the observer a little early, compared to that from the star at the far end. In case of perfectly eclipsing binary stars, the light from the star at the far end should actually have to travel extra distance, which is equal to the major axis between the two stars. This will be considerable when the distance between two stars is more. The line of sight errors have been calculated and tabulated for five binary stars.
\end{abstract}

Key words: binary stars - cosmogony - binary stars - eclipsing

\section{Introduction}

Let us consider perfectly eclipsing binary stars. It is shown in figure 1 . In this diagram S1 and S2 are the binary stars. For illustrating the concept, let us assume that the stars S1 and S2 are of same mass and same sizes, rotating in a perfectly circular orbit, with the orbit orientation $90^{\circ}$ to the plane of the observer.This means that there will be a perfect eclipse between the stars.Since $\mathrm{S} 1$ and $\mathrm{S} 2$ are identical in all respects, they will be rotating at the same distance from the common center "O", with same radial velocity.

In the position shown in figure 1, the velocities calculated by spectroscopic observations of blue shift and red shift will be exactly equal, as the light from $\mathrm{S} 1$ and S2 reach the observer at the same time. In all the binary star figures considered here, the direction of rotation of stars is assumed as counter clockwise direction, as shown by the arrow mark.

When the stars are at slightly different position after moving through an angle $\alpha$, as shown in figure 2, the lights from S1 and S2 will not reach the observer at the same time. In this position, S2 is nearer to the observer than S1. Also $\mathrm{S} 2$ is travelling towards observer whereas $\mathrm{S} 1$ is moving away from the observer. Therefore, light from S2 will be blue shifted and that from S1 will be red shifted. 


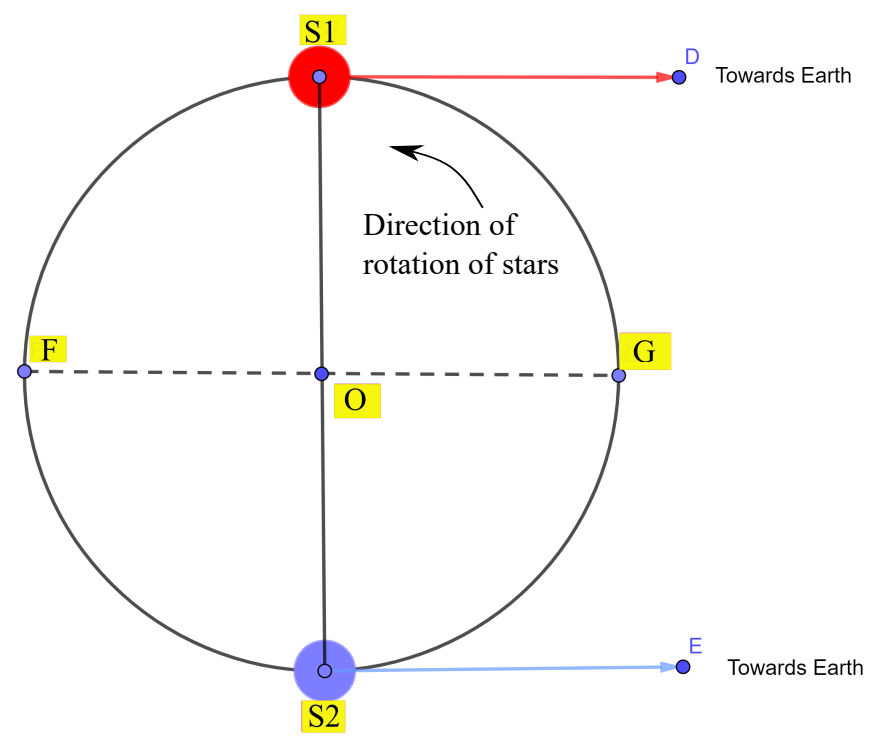

Figure 1. Stars at equal distance from the observer

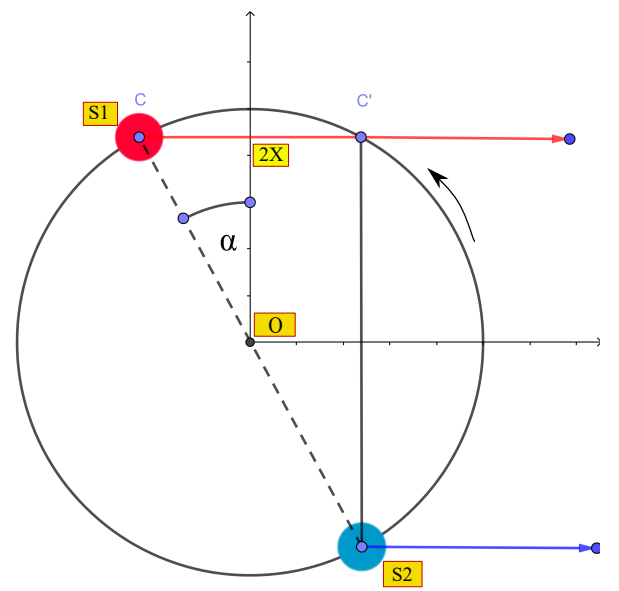

Figure 2. Stars at different distances from the observer

But the important observation to be made here is that the light coming from $\mathrm{S} 1$ has to travel a longer distance compared to that from S2. This extra distance is denoted as $2 \mathrm{x}$ in the figure 2 .

Therefore it will to take a longer time in reaching the observer. This has to be taken into account when dealing with stars with considerable distance 
between them with long periods (Kniazev, 2020), in which case the period of light travelling through a distance of $2 \mathrm{X}$ will be considerable. As an example, if the light takes 100 minutes to travel $2 \mathrm{X}$ distance, then the spectrum of S1 is to be observed after 100 minutes compared to the spectrum of S2. That means, there should be a gap of 100 minutes between the matching spectrums of which we actually have to consider for various calculations. Such time errors are calculated for five different types of binary stars.

\subsection{Theoretical positions of stars}

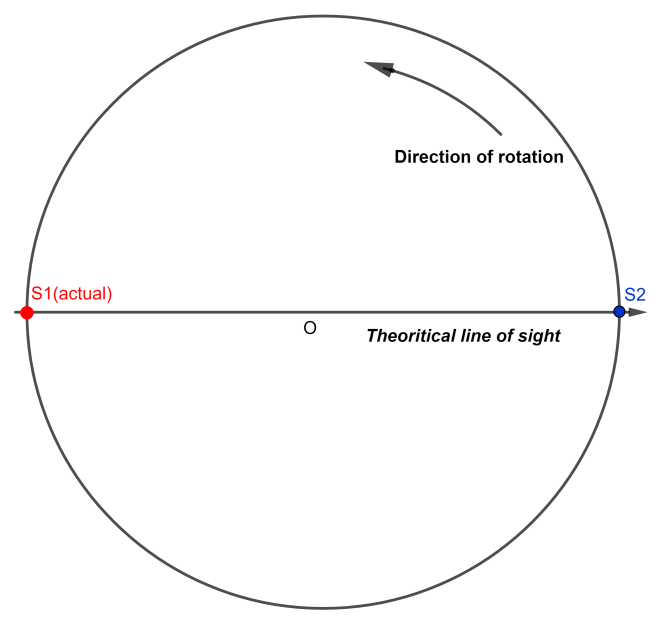

Figure 3. Theoretical positions of stars at eclipse

As indicated in figure 3, theoretical positions of S1and S2 are along the line of common centre "O". Figure 3 shows the positions of S1 and S2 at complete eclipse state. Directions of motion of stars are counter clockwise, as indicated by arrow mark.

\subsection{Actual positions of stars}

As indicated in figure 4, light from S1 has to travel almost a distance equal to major axis (2a) compared to the light from S2. During this time, stars will have moved through some distance. Hence, the star S2 will obstruct light emitted by $\mathrm{S} 1$ when S1 was at the apparent position, shown by dotted line in figure-4.

Figure 4 shows the positions of S1 and S2 at complete eclipse state. Even at 


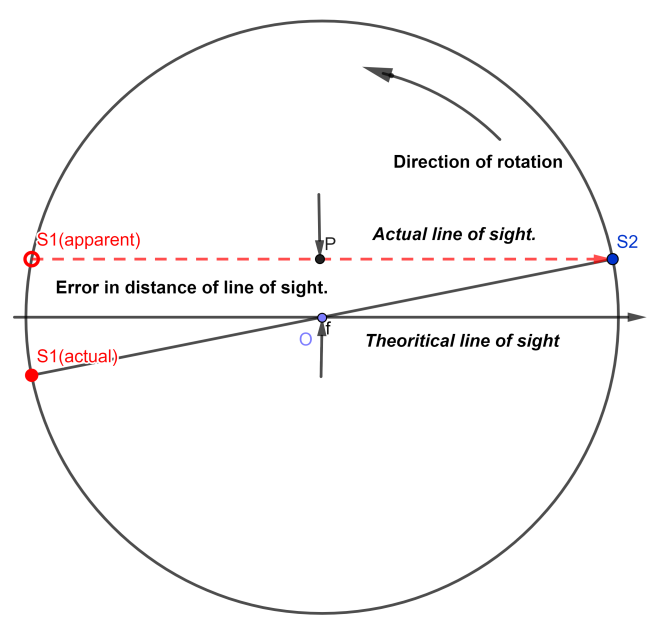

Figure 4. Actual positions of stars at eclipse

this stage, the stars will be along apparent line of sight and not along the theoretical line of sight. So it is evident that the eclipse is taking place to the observer when the stars are not actually in line of sight of the observer. The error distance between actual line of sight and theoretical line of sight is indicated in the figure.

\subsection{Equations and calculations}

Time taken for the light to travel " $2 \mathrm{x}$ " distance as shown in figure- 2 is given by:

$$
t 1=2 x / c .
$$

where " $\mathrm{t} 1$ " is the time taken for light to travel through a distance $=2 \mathrm{x}$ and "c" is the velocity of light. This may me negligible for many binaries with very low periods of rotations but will be considerable in case of stars with long periods of revolutions.But in short period binary stars, angular errors will be more.

This time delay will be maximum when the stars are at extreme positions as shown in the figure 3. So, " $2 \mathrm{x}$ " will now be equal to" $2 \mathrm{a}$ "

$$
\text { tmaxe }=2 a / c .
$$

where:

"tmaxe" is the time taken for light to travel through a distance $=2 \mathrm{a}$,

"a" is the notation used to represent semi major axis $=$ half of the distance between two stars, 
and "c" is the velocity of light

During this time tmaxe, the stars will travel through a distance "De". This is given by

$$
D e=\text { tmaxe } *(V o)
$$

where "Vo" is the orbital velocity of stars

From equation (2) and (3), we get

$$
D e=2 a *(V o) / c
$$

Error can be calculated in terms of angle in degrees also, with the parameters as shown in the figure 4. Here, the stars have moved through an arc length "De". Since the angle is very small, the curved distance traversed by the planet is approximated to linear distance The angle subtended is denoted as $\theta$.

Hence it is calculated by

$$
\theta=\sin ^{-1}[D e] / a
$$

Substituting for "De" using equation (4), we get

$$
\theta=\sin ^{-1} \frac{[2 *(V o)]}{c}
$$

The stars will cover the circumference in a time period "P". The length of the circumference is given by:

$$
\text { Circumference }=C=2 a \pi
$$

The circumference and period are related by the expression:

$$
C=\left(V_{o} *\right) P
$$

where "Vo" is the radial velocity in $\mathrm{km} / \mathrm{sec}$ and "P" is the period in seconds.

From equations (7) and (8), we have:

$$
V o * P=\frac{2 a}{\pi}
$$

Hence semi major axis is given by:

$$
a=\frac{V o * P}{2 \pi}
$$

Substituting this in equation(2), we get

$$
\text { tmaxe }=\frac{V o * P}{\pi * c}
$$

Since " $\pi$ " and "c" are constants, tmaxe is proportional to the product of orbital velocity and period. The input parameters for the above calculation of distance errors are taken from research paper articles. (Deb \& Singh, 2011) 


\section{INTERESTING EXAMPLES}

Here, five spectroscopic eclipsing binaries(Christiansen et al., 2007) of short period,(Torres et al., 2020) little long period, long period(Maxted \& Hutcheon, 2018) and very long period are considered. Error (Mighell \& Plavchan, 2013) calculations are done by using equation (2) for maximum time errors and equation (4) for distance calculation. Most of the input data have been taken from kepler catalog (Kirk et al., 2016).

\subsection{Very short period binary-ZTF j1539}

It is an eclipsing spectroscopic neutron binary star system, with a period of 6.91 minutes (0.00479 days approx.)(Littenberg \& Cornish, 2019) The stars are separated by only about $78000 \mathrm{~km}$. The orbital velocity of main star is very high and is $591 \mathrm{~km} / \mathrm{s}$.

The error time at maximum position is 0.26 seconds. Error in distance from the line of sight $=153.66 \mathrm{~km}$.

\subsection{Short period binary-Algol}

It is an eclipsing spectroscopic binary star system, with a period of 69 hours and 49 minutes (2.8673 days approx.) The stars are separated by about 7.5 million $\mathrm{Km}(0.05 \mathrm{AU})$. One major and one minor eclipse occur in a cycle. The orbital velocity of main star is $95.1 \mathrm{~km} / \mathrm{s}$.

The error time at maximum position is 25 seconds. Error in distance from the line of sight $=2377 \mathrm{~km}$.

\section{3. little long period binary-Beta Lyrae}

It is also eclipsing spectroscopic binary star system,(Linnell, 2000) with a comparatively long period of 12.89 days approx. The stars are separated by about $17725019 \mathrm{~km}$. The orbital velocity of main star is $50 \mathrm{~km} / \mathrm{s}$.

The error time at maximum position is 59.08 seconds. Error in distance from the line of sight $=2954.2 \mathrm{~km}$.

\section{4. long period binary-WOCS 23009 (A851)}

It is a totally eclipsing binary star system,(Sandquist et al., 2012) with a very long period of 771.8 days approx. The stars are almost perfectly in plane of the observer. The mass ratio of two stars are 1.23:1. The stars are separated by about $141998000 \mathrm{Km}$. The orbital velocity of main star is $6.69 \mathrm{~km} / \mathrm{s}$.

The error time at maximum position is 473.3 seconds. Error in distance from the line of sight $=3166 \mathrm{~km}$. 


\subsection{Very long period binary-Hd54662}

It is an eclipsing spectroscopic binary star system, with a very long period of 2103.4 days (approx).(Mossoux et al., 2018) The stars are separated by about $1143800000 \mathrm{Km}$. The orbital velocity of main star is $97 \mathrm{~km} / \mathrm{s}$.

The error time at maximum position is 3812.7 seconds (63.55 minutes approx.). Error in distance from the line of sight $=369828.6 \mathrm{~km}$.

\section{Results and discussions}

All the important parameters and errors are shown in table-1.

Table 1. Details of important parameters and calculated errors.

\begin{tabular}{|c|c|c|c|c|c|}
\hline \hline Star name & Period $(\mathrm{d})$ & $\mathrm{Vo}(\mathrm{km} / \mathrm{sec})$ & $2 \mathrm{a}(\mathrm{Kkm})$ & $\mathrm{De}(\mathrm{km})$ & tmaxe $(\mathrm{sec})$ \\
\hline ZTF J1539 & 0.00479 & 591 & 78000 & 153.66 & 0.26 \\
\hline Algol & 2.87 & 95.1 & 7500 & 2377.5 & 25 \\
\hline Beta Lyrae & 12.89 & 50 & 17725 & 2954.2 & 59.08 \\
\hline WOCS23009 & 771.8 & 6.69 & 141998 & 3166.6 & 473.3 \\
\hline Hd54662 & 2103.4 & 97 & 1143800 & 369828.6 & 3812.7 \\
\hline
\end{tabular}

Note: here "Vo" is orbital velocity in $\mathrm{Km} / \mathrm{sec}$,"2a" is the length of the major axis in Kkm, which is thousand km,"De" is the distance error in Km and "tmaxe" is the spectrum time delay error in seconds.

\subsection{Time errors between stars in a binary.}

It is very evident that the time errors increase with the period and radial velocity . For longest period binary star Hd54662, we are having 63.55 minutes of error. This means that we have to consider the red shift of S1 after 63.55 minutes,from the blue shift time of $\mathrm{S} 2$, to get the exact phase positions of stars, as shown in figure 4. If we simply take the redshift and blueshift at the same time, we will get the phase error of 63.55 minutes. This can be done in space based telescopes.

\subsubsection{Problem with ground based telescopes}

In ground based telescopes, if we take spectrum of star 1 after 63.55 minutes to that of star 2, then the errors due to atmospheric refraction and density variations along with gravitational field variations will be more after 63.55 minutes. So, there is no alternative other than taking spectrum of both stars at the same time and then apply corresponding corrections to get the accurate results. Note: Period of revolution will not be affected by this error, as it is found by interval between two eclipses. 


\subsection{Line of sight distance errors}

The line of sight error is obviously proportional to the period and orbital velocity of stars. The distance error for ZTF J1539 is $153.66 \mathrm{Km}$.It is the highest for the binary having maximum period. that is HD54662 witha period of 2103.4 days is $369828.6 \mathrm{Km}$.

\subsection{Concluding remarks}

This paper shows that stars appear to move unequal distances in equal interval of time. Figure- 5 shows that the red shifted portion is $179.77^{\circ}$ and blue shifted

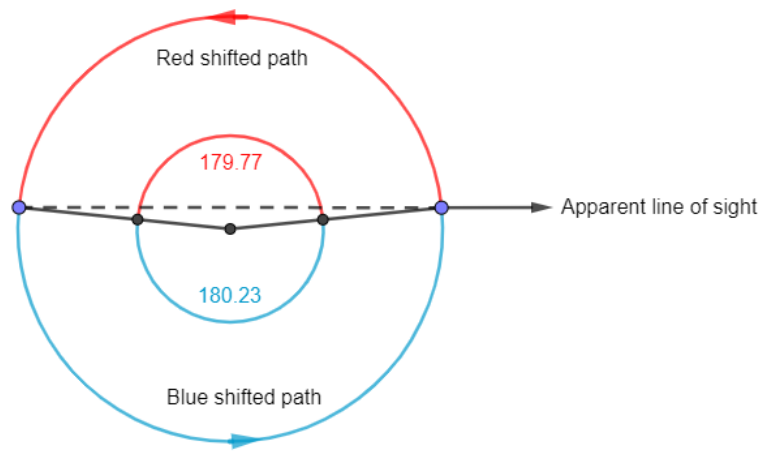

Figure 5. Red shift and blue shift paths.

portion is $180.23^{\circ}$ for ZTF J1539 binary star. This means that stars appear to cover unequal distances in equal intervals of time. When viewed edge-on, it should appear like the one shown in figure -6 .

With very high resolution telescope, the observer must be able to see that blue

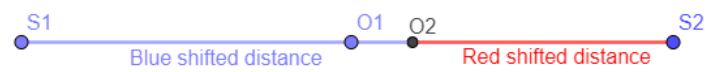

Figure 6. Edge-on view of paths.

shifted length is little more than the red shifted length, but stars are covering these unequal distances in equal intervals of time. But the problem is that the 
present day telescopes are unable to even resolve that there are two stars in many binaries. Astronomers are finding them by spectroscopic analysis. (Lehmann et al., 2012)

This effect is not just for binary stars.If a distant observer see our Milky way galaxy edge-on, he will see that each star at the edge of the galaxy will cover $179^{\circ}$ red shifted $181^{\circ}$ blue shifted paths, but covered in equal intervals of time. Distance error increases with increase in orbital period.Due to this error, the observer will be seeing eclipsing in apparent line of sight.

\section{References}

Christiansen, J., Derekas, A., Ashley, M., Webb, J., Hidas, M., Hamacher, D. W., \& Kiss, L. (2007). The first high-amplitude $\delta$ scuti star in an eclipsing binary system. Monthly Notices of the Royal Astronomical Society, 382(1), $239-244$.

Deb, S., \& Singh, H. P. (2011). Physical parameters of 62 eclipsing binary stars using the all sky automated survey-3 data- i. Monthly Notices of the Royal Astronomical Society, 412(3), 1787-1803.

Kirk, B., Conroy, K., Prša, A., Abdul-Masih, M., Kochoska, A., MatijeviČ, G., ... others (2016). Kepler eclipsing binary stars. vii. the catalog of eclipsing binaries found in the entire kepler data set. The Astronomical Journal, $151(3), 68$.

Kniazev, A. (2020). Long-period eclipsing binaries: towards the true massluminosity relation. ii. absolute parameters of the nn del system. Astrophysics and Space Science, 365(10), 1-12.

Lehmann, H., Zechmeister, M., Dreizler, S., Schuh, S., \& Kanzler, R. (2012). Kic 4247791: a sb4 system with two eclipsing binaries (2ebs)-a quadruple system? Astronomy \& Astrophysics, 541, A105.

Linnell, A. (2000). Progress on a model for $\beta$ lyrae. Monthly Notices of the Royal Astronomical Society, 319(1), 255-266.

Littenberg, T. B., \& Cornish, N. J. (2019). Prospects for gravitational wave measurement of ztf j1539+ 5027. The Astrophysical Journal Letters, $881(2)$, L43.

Maxted, P., \& Hutcheon, R. (2018). Discovery and characterisation of longperiod eclipsing binary stars from kepler $\mathrm{k} 2$ campaigns 1,2 , and 3 . Astronomy E Astrophysics, 616, A38.

Mighell, K. J., \& Plavchan, P. (2013). Period error estimation for the kepler eclipsing binary catalog. The Astronomical Journal, 145(6), 148.

Mossoux, E., Mahy, L., \& Rauw, G. (2018). The long-period massive binary hd 54662 revisited. Astronomy \& Astrophysics, 615, A19. 
Sandquist, E. L., Mathieu, R. D., Brogaard, K., Meibom, S., Geller, A. M., Orosz, J. A., ... others (2012). A long-period totally eclipsing binary star at the turnoff of the open cluster ngc 6819 discovered with kepler. The Astrophysical Journal, 762(1), 58.

Torres, G., Vanderburg, A., Curtis, J. L., Kraus, A. L., Rizzuto, A. C., \& Ireland, M. J. (2020). Eclipsing binaries in the open cluster ruprecht 147. iii. the triple system epic 219552514 at the main-sequence turnoff. The Astrophysical Journal, 896(2), 162. 